\title{
Sodium nitrite attenuates hypertension-in-pregnancy and blunts increases in soluble fms-like tyrosine kinase- 1 and in vascular endothelial growth factor
}

\author{
Victor Hugo Gonçalves-Rizzi, Jose Sergio Possomato-Vieira, Tamiris Uracs Sales Graça, \\ Regina Aparecida Nascimento, Carlos A. Dias-Junior* \\ Department of Pharmacology, Biosciences Institute of Botucatu, Sao Paulo State University (UNESP), Botucatu, Sao Paulo, Brazil
}

\section{A R T I C L E I N F O}

\section{Article history:}

Received 6 March 2016

Received in revised form

5 May 2016

Accepted 11 May 2016

Available online 12 May 2016

\section{Keywords:}

Hypertension-in-pregnancy

Sodium nitrite

$\mathrm{N}(\mathrm{G})$-nitro-L-arginine methyl ester

Rats

\begin{abstract}
A B S T R A C T
Preeclampsia is a pregnancy-associated disorder characterized by hypertension with uncertain pathogenesis. Increases in antiangiogenic soluble fms-like tyrosine kinase-1 (sFlt-1) and reductions in nitric oxide (NO) bioavailability have been observed in preeclamptic women. However, the specific mechanisms linking these detrimental changes to the hypertension-in-pregnancy are not clearly understood. In this regard, while recent findings have suggested that nitrite-derived NO formation exerts antihypertensive and antioxidant effects, no previous study has examined these responses to orally administered nitrite in hypertension-in-pregnancy. We then hypothesized restoring NO bioavailability with sodium nitrite in pregnant rats upon NO synthesis inhibition with N(omega)-nitro-L-arginine methyl ester (LNAME) attenuates hypertension and high circulating levels of sFlt-1. Number and weight of pups and placentae were recorded to assess maternal-fetal interface. Plasma sFlt-1, vascular endothelial growth factor (VEGF) and biochemical determinants of NO formation and of antioxidant function were measured. We found that sodium nitrite blunts the hypertension-in-pregnancy and restores the NO bioavailability, and concomitantly prevents the L-NAME-induced high circulating sFlt- 1 and VEGF levels. Also, our results suggest that nitrite-derived NO protected against reductions in litter size and placental weight caused by L-NAME, improving number of viable and resorbed fetuses and antioxidant function. Therefore, the present findings are consistent with the hypothesis that nitrite-derived NO may possibly be the driving force behind the maternal and fetal beneficial effects observed with sodium nitrite during hypertension-in-pregnancy. Certainly further investigations are required in preeclampsia, since counteracting the damages to the mother and fetal sides resulting from hypertension and elevated sFlt- 1 levels may provide a great benefit in this gestational hypertensive disease.
\end{abstract}

(c) 2016 Elsevier Inc. All rights reserved.

\section{Introduction}

Preeclampsia and related hypertensive pregnancy disorders affect $5-8 \%$ of all births in the United States, resulting in $15-20 \%$ of maternal deaths worldwide [1]. These disorders present serious complications to the mother and the baby, and the mechanisms involved are not clearly understood [2]. Currently, despite intense investigation, definitive treatment is limited to preterm delivery of

\footnotetext{
* Corresponding author. Department of Pharmacology, Biosciences Institute of Botucatu, Sao Paulo State University, Distrito de Rubiao Junior, S/N, 18.618-970 Botucatu, SP, Brazil.

E-mail address: carlosjunior@ibb.unesp.br (C.A. Dias-Junior).
}

the baby and placenta, suggesting that the causative symptoms of preeclampsia may be dependent on the presence of the placenta [3]. In fact, placental ischemia is thought to be an initiating event, leading to the release of circulating biomarkers of inflammatory response, oxidative stress and antiangiogenic factors in the maternal circulation $[4,5]$.

There may be an imbalance among the pro- and antiangiogenic factors, in which the circulating antiangiogenic protein sFlt-1 (soluble fms-like tyrosine kinase-1) binds and sequesters the vascular endothelial growth factor (VEGF), causing endothelial dysfunction and producing preeclampsia-like symptoms [6]. Accordingly, the extracorporeal removal of circulating sFlt-1 in preeclamptic patients may improve the symptoms [7], thus, confirming the key role of sFlt-1 in the maternal side of the disorder. 
However, it has been shown that the high levels of circulating sFlt-1 and uteroplacental circulation are not affected by the most commonly antihypertensive drugs used in clinic to treat preeclampsia $[2,4,6]$. In addition, reductions on the formation of nitric oxide (NO) may be inversely related to serum levels of sFlt-1, highlighting that hypertension-in-pregnancy during preeclampsia may be explained, at least in part, by reductions of NO bioavailability [8-10].

Importantly, accumulated experimental evidences have showed a potential role for the anion nitrite, being more than a simple biomarker of NO formation. Nitrite is recycled back to NO as a physiological alternative to NO formation independent of NO synthase (NOS)-related pathways, restoring the vasodilator actions of the NO [11-13]. In this context, recent studies have suggested that NO generation from nitrite may occur in conditions such as hypoxia and that sodium nitrite may selectively deliver NO to ischemic/ hypoxic tissues [12,13]. Therefore, if uteroplacental ischemia is thought to play a major role in preeclampsia [4,5], we hypothesized that these conditions would create the ideal biochemical environment for the in vivo reduction of nitrite to NO, thus, attenuating hypertension (mother side) and concomitantly improving fetal detrimental changes caused by hypertension-in-pregnancy.

In order to confirm this hypothesis, pregnant rats were treated with N(omega)-nitro-L-arginine methyl ester (L-NAME), an inhibitor of NO synthesis, during mid-to late gestation, in which hypertension, reductions of litter size [14] and placental weight [15] are manifested. We have also examined the circulating levels of sFlt-1 and VEGF, and biochemical determinants of oxidative stress [16], and if the attenuation of hypertension with sodium nitrite would be associated with reduced levels of sFlt- 1 and antioxidant effects [11].

\section{Materials and methods}

\subsection{Animals and experimental protocol}

Wistar rats $(200-250 \mathrm{~g})$ were housed in cages at $22 \pm 2{ }^{\circ} \mathrm{C}$ on a 12-hr light/dark cycle and given free access to water and rat chow. Each female rat was separately mated overnight. Day 0 of pregnancy was defined as the day when spermatozoa were found in a vaginal smear.

On pregnancy day 14, each pregnant rat mother was first placed into a single cage and randomized to one of the four treatment groups ( $\mathrm{n}=10$ per group, total of 40 rats): Norm-Preg, Preg + Sodium nitrite, HTN-Preg and HTN-Preg + Sodium nitrite groups. Pregnant rats received daily $0.9 \%$ saline solution by gavage and by via intraperitoneal (i.p.) in Norm-Preg group; or sodium nitrite by gavage (Sodium nitrite; Sigma, St. Louis, MO, \#S2252; $15 \mathrm{mg} / \mathrm{kg} /$ day for 7 days) and saline injections by via i.p. in Preg + Sodium nitrite group; or i.p. injections of N(G)-nitro-Larginine methyl ester (L-NAME; Sigma, St. Louis, MO, \# 5751; $60 \mathrm{mg} / \mathrm{kg} /$ daily [17]) and saline solution by gavage in HTN-Preg group; or i.p. injections of L-NAME (60 mg/kg/daily) and sodium nitrite by gavage ( $15 \mathrm{mg} / \mathrm{kg} /$ day for 7 days) in HTN-Preg + Sodium nitrite group.

The dose of sodium nitrite $(15 \mathrm{mg} / \mathrm{kg}$ or $0.217 \mathrm{mmol} / \mathrm{kg}$; by gavage) was chosen with basis on previous studies showing that this dose exerts relevant antihypertensive and antioxidant effects in rats $[11,18-21]$.

Rats were euthanized on gestation-day 21 under overdose of isoflurane followed by exsanguination. Blood samples were collected in lyophilised ethylenediaminetetraacetic acid (EDTA) (Vacuntainer Becton-Dickinson, BD, Oxford, UK) and immediately centrifuged and plasma was separated and stored at $-80{ }^{\circ} \mathrm{C}$ until use for biochemical analysis.

All procedures for animal experimentation were approved by the Ethics Committee, Biosciences Institute of Botucatu, State University of Sao Paulo (Protocol \#618/2014), which is complied with international guidelines of the European Community for the use of experimental animals.

\subsection{Blood pressure measurements}

Systolic blood pressure ( $\mathrm{mmHg}$ ) was measured on gestational day 13 (baseline with absence of gavage or i.p. injections) and days 14, 16, 18 and 20, $6 \mathrm{~h}$ after drugs administration, using tail-cuff plethysmography (Insight, Ribeirao Preto, Sao Paulo, Brazil, \# EFF 306). Briefly, all pregnant rats were first acclimated in a quiet room, conditioned and restrained for 5-10 min in a warm box (Insight, Ribeirao Preto, Sao Paulo, Brazil, \# EFF-307) to the measurements for 3 days before the pregnancy day 14 (these data were discarded) and then the baseline systolic blood pressure was determined as the average of the cuff inflation-deflation (3-6) cycles by a trained operator on pregnancy day 14 [22].

\subsection{Effects on placenta and fetuses}

On gestational-day 21, after euthanasia, animals were placed in supine position and cesarean section was performed. The averages of total number of viable fetuses, litter size, fetal weight and placental weight of each mother were recorded. Viable fetuses were determined as those which showed no macroscopical sign of malformation and could apparently have a normal outcome with the progression of the pregnancy, as previously reported [23].

\subsection{Measurement of plasma NOx (nitrate + nitrite) concentrations}

The plasma NOx concentrations were determined in duplicate by using the Griess reaction, as previously described [24]. Briefly, $40 \mu \mathrm{L}$ of plasma were incubated with the same volume of nitrate reductase buffer $(0.1 \mathrm{M}$ potassium phosphate, $\mathrm{pH} 7.5$, containing $1 \mathrm{mM} \beta$-nicotinamide adenine dinucleotide phosphate and $2 \mathrm{U}$ of nitrate reductase $/ \mathrm{mL}$ ) in individual wells of a 96 -well plate. Samples were allowed to incubate overnight at $37^{\circ} \mathrm{C}$ in the dark; $8 \mu \mathrm{L}$ of freshly prepared Griess reagent ( $1 \%$ sulfanilamide, $0.1 \%$ naphthylethylenediamine dihydrochloride in $5 \%$ phosphoric acid) were added to each well and the plate was incubated, for 15 additional minutes, at room temperature. A standard nitrate curve was obtained by incubating sodium nitrate $(0.2-200 \mu \mathrm{M})$ with the same reductase buffer. The NOx levels in plasma were expressed in $\mu \mathrm{mol} /$ L.

\subsection{Determination of sFlt-1 and VEGF}

Commercial enzyme immunoassay (ELISA) kits for sFlt-1 $(R \& D$ Systems Inc, Minneapolis, MN, USA \#MVR100) and VEGF (R\&D Systems Inc, Minneapolis, MN, USA \#RRV00) were used to determine plasma levels. Assays were performed according to manufacturer's instructions. Plasmatic levels of sFlt-1 and VEGF were expressed in $\mathrm{pg} / \mathrm{mL}$.

\subsection{Determination of myeloperoxidase (MPO) activity}

Circulating plasma levels of MPO reflect the inflammatory response, as according to the method previously proposed by Suzuki [25]. Briefly, $30 \mu \mathrm{L}$ of centrifuged plasma samples received $100 \mu \mathrm{L}$ of TMB (tetramethyl benzidine) and $0.04 \%$ of $\mathrm{H}_{2} \mathrm{O}_{2}$. Posteriorly, microplate was incubated for $10 \mathrm{~min}$ at $37^{\circ} \mathrm{C}$, protected from light. The reaction was stopped with $100 \mu \mathrm{L}$ of $\mathrm{H}_{2} \mathrm{SO}_{4}(2 \mathrm{~N})$ and the absorbance at $450 \mathrm{~nm}$ with correction to $630 \mathrm{~nm}$ was read with the spectrophotometer (Synergy 4, BIOTEK, Winooski, VT, USA). The 
results were expressed in $\Delta A_{630} / \mathrm{min} / \mathrm{mL}$ ).

\subsection{Measurements of plasma antioxidant capacity}

The trolox equivalent antioxidant capacity (TEAC) was performed as previously described [26]. Briefly, a standard curve was established using $100 \mu \mathrm{g}$ of Trolox (6-hidroxy-2,5,7,8 - tetramethylchroman-2-carboxylic-acid, Sigma, St. Louis, MO, USA, catalogue\# 238813) in $1 \mathrm{~mL}$ of sodium acetate buffer $(0.4 \mathrm{M}$, $\left.\mathrm{C}_{2} \mathrm{H}_{3} \mathrm{NaO}_{2} \cdot 3 \mathrm{H}_{2} \mathrm{O}\right)+$ glacial acetic acid $(0.4 \mathrm{M})$. Firstly, $20 \mu \mathrm{L}$ of plasma samples were added to $200 \mu \mathrm{L}$ of sodium acetate buffer + glacial acetic acid and the absorbance at $660 \mathrm{~nm}$ was read with the spectrophotometer (Synergy 4, BIOTEK, Winooski, VT, USA). Secondly, $20 \mu \mathrm{L}$ of sodium acetate buffer $(0.03 \mathrm{M})$ and glacial acetic acid $(0.03 \mathrm{M})+\mathrm{H}_{2} \mathrm{O}_{2}+$ ABTS (2,2'-azino-bis(3-ethylbenzthiazolin-6 sulfonic acid, Sigma A 1888) was added to the samples and incubated for 5 min. Finally, a second spectrophotometer read was performed at $660 \mathrm{~nm}$. The second reading values were subtracted from the values found in the first reading and the antioxidant activity of the sample was expressed as mmol of Trolox equivalent/L.

\subsection{Evaluation of the antioxidant status of plasma}

Direct reductions of MTT (3-(4,5-dimethylthiazol-2-yl)-2,5diphenyltetrazolium bromide), Sigma, St Louis, MO, USA) were measured as previously described [27]. Briefly, $100 \mu \mathrm{L}$ of plasma were mixed with $12.5 \mu \mathrm{l}$ of dye solution ( $5 \mathrm{mg} / \mathrm{mL}$ in PBS); the final volume was adjusted to $200 \mu \mathrm{l}$ with PBS and the mixture was incubated for $60 \mathrm{~min}$ at $37^{\circ} \mathrm{C}$. The reaction was terminated by the addition of $750 \mu \mathrm{L}$ of $0.04 \mathrm{M}$ hydrochloric acid in isopropanol. The tubes were centrifuged for $10 \mathrm{~min}$ at $1000 \times \mathrm{g}$ and the supernatant collected and the absorbance were measured at $570 \mathrm{~nm}$.

\subsection{Statistical analysis}

Using commercially available statistical software (Graph Pad Prism $^{\mathbb{B}}$ 6.0 for Windows, San Diego, CA), a Shapiro-Wilk test was applied to verify normality of data distribution. Systolic blood pressure measurements were submitted to a two-way analysis of variance (ANOVA) with time and treatment defined as main effects, followed by Bonferroni's correction for multiple comparisons among groups to compare measurements on pregnancy days 16,18 and 20, or one-way ANOVA followed by Bonferroni's correction for multiple comparisons were used to compare fetal and placental changes or sFlt-1, VEGF, TEAC, MPO, MTT and NOx levels. Since only fetal weight in Norm-Preg group presented no normal distribution, the Mann-Whitney test was used to compare the fetal weight among the four groups. Statistical significance was considered at $\mathrm{P}<0.05$. All values are expressed as mean \pm SEM.

\section{Results}

There were no significant differences in the systolic blood pressure values $(116-123 \pm 4 \mathrm{mmHg}$ ) among the four groups on days 13 and $14(P>0.05$, Fig. 1$)$. Systolic blood pressure values were elevated in the HTN-Preg group on days 16, 18 and 20 (145 \pm ; $149 \pm 3$ and $140 \pm 4 \mathrm{mmHg}$, respectively, ${ }^{*} P<0.05$, Fig. 1 ). However, HTN-Preg + Sodium nitrite diminished systolic blood pressure values were significantly observed on days 16,18 and 20 (126 \pm 3 ; $131 \pm 3$ and $127 \pm 2.3 \mathrm{mmHg}$ ) compared to HTN-Preg group $\left({ }^{\#} P<0.05\right.$, Fig. 1$)$.

Number of pups (litter size) was significantly lower in HTN-Preg $(9.2 \pm 0.7)$ if compared to Norm-Preg, Preg + Sodium nitrite or HTN-Preg + Sodium nitrite groups $(13 \pm 1 ; 11 \pm 0.6$ or $12 \pm 0.5$, respectively), ${ }^{*} P<0.05$, Fig. $\left.2 A\right)$. Also, lower numbers of viable fetuses with higher number of resorbed fetuses were found only in HTN-Preg $(7 \pm 0.7$ and $1.20 \pm 0.29$, respectively) and were compared to Norm-Preg, Preg + Sodium nitrite and HTNPreg + Sodium nitrite groups $(11 \pm 1.0$ and $0.30 \pm 0.15 ; 11 \pm 0.7$ and $0.20 \pm 0.13$, and $11 \pm 0.6$ and $0.40 \pm 0.16$, respectively, ${ }^{*} P<0.05$, Fig. 2B and C). Surprisingly, the fetal weight was significantly higher only in the Preg + Sodium nitrite group $(3.9 \pm 0.05 \mathrm{~g})$ if compared to Norm-Preg, HTN-Preg and HTN-Preg + Sodium nitrite groups $\left(2.9 \pm 0.1 ; 3.1 \pm 0.03\right.$ and $3.1 \pm 0.04 \mathrm{~g}$, respectively, ${ }^{\#} P<0.05$, Fig. 2D). Lower placental weight was found only in HTN-Preg group $(0.53 \pm 0.01 \mathrm{~g})$ if compared to Norm-Preg, Preg + Sodium nitrite and HTN-Preg + Sodium nitrite groups $(0.63 \pm 0.01 ; 0.6 \pm 0.01$ and $0.6 \pm 0.01$ g respectively, ${ }^{*} P<0.05$, Fig. $2 \mathrm{E}$ ).

We evaluated NO bioavailability by measuring plasma nitrite and nitrate concentrations. We found significant lower NO bioavailability in HTN-Preg group $(49 \pm 7 \mu \mathrm{mol} / \mathrm{L})$ compared to those found in the Norm-Preg group $\left(72 \pm 8 \mu \mathrm{mol} / \mathrm{L},{ }^{*} \mathrm{P}<0.05\right.$, Fig. 3A). However, the treatment with sodium nitrite enhanced the NO bioavailability in both Preg + Sodium nitrite and HTNPreg + Sodium nitrite groups $(138 \pm 10$ and $131 \pm 11 \mu \mathrm{mol} / \mathrm{L}$, respectively, ${ }^{\#} P<0.05$, Fig. $3 \mathrm{~A}$ ). Also, no significant differences were found in NO bioavailability between Preg + Sodium nitrite and HTN-Preg + Sodium nitrite groups $(P>0.05$, Fig. $3 A)$.

Sodium nitrite blunted both increases in free sFlt-1 and VEGF levels induced by L-NAME (both ${ }^{*} P<0.05$, Fig. 3B and C). Increasedfree sFlt-1 levels were observed only in HTN-Preg group, if compared with Norm-Preg, Preg + Sodium nitrite and HTNPreg + Sodium nitrite groups $(368 \pm 48 ; 162.2 \pm 4.3 ; 136 \pm 9$ and $190 \pm 22 \mathrm{pg} / \mathrm{mL}$ respectively, ${ }^{*} P<0.05$, Fig. 3B). Higher levels of VEGF in plasma were found only in HTN-Preg group, if compared to Norm-Preg, Preg + Sodium nitrite and HTN-Preg + Sodium nitrite groups $(77 \pm 4.6 ; 58 \pm 6 ; 57 \pm 3$ and $62 \pm 1 \mathrm{pg} / \mathrm{mL}$ respectively, ${ }^{*} \mathrm{P}<0.05$, Fig. $\left.3 \mathrm{C}\right)$.

Lower anti-oxidant capacity was found only in HTN-Preg group $(0.04 \pm 0.01)$ if compare to Norm-Preg, Preg + Sodium nitrite or HTN-Preg + Sodium nitrite groups $(0.11 \pm 0.01 ; 0.13 \pm 0.01$ and $0.09 \pm 0.01$, respectively, ${ }^{*} P<0.05$, Fig. 4 A). Both Preg + Sodium nitrite and HTN-Preg + Sodium nitrite groups presented increases in antioxidant status of plasma $(0.131 \pm 0.002$ and $0.130 \pm 0.002$, respectively) if compared to control Norm-Preg and HTN-Preg groups $\left(0.11 \pm 0.009\right.$ and $0.11 \pm 0.006$, respectively, ${ }^{\#} P<0.05$, Fig. 4B).

Also, HTN-Preg and HTN-Preg + Sodium nitrite groups presented increases in myeloperoxidase levels (2.06 \pm 0.10 and $1.9 \pm 0.15$, respectively) if compared to Norm-Preg and Preg + Sodium nitrite groups ( $1.12 \pm 0.2$ and $1.13 \pm 0.2$, respectively, ${ }^{* *} P<0.05$, Fig. 4C).

\section{Discussion}

This study shows that sodium nitrite, administered orally and once a day, diminishes systolic blood pressure in hypertension-inpregnancy induced by L-NAME and concomitantly prevents the increases in circulating plasma sFlt-1 and VEGF levels. Also, our results suggest that increases in nitrite-derived $\mathrm{NO}$ has protected against reductions in number of viable fetuses, litter size and placental weight caused by L-NAME. In addition, we observed increases in MPO levels with concomitant reductions in antioxidant function of plasma induced by L-NAME, suggesting presence of oxidative stress in this rat model of hypertension-in-pregnancy. Also, sodium nitrite has maintained the antioxidant function in hypertensive pregnant rats. Therefore, the present findings are consistent with the idea that treatment with sodium nitrite attenuates the hypertension-in-pregnancy and that nitrite-derived 


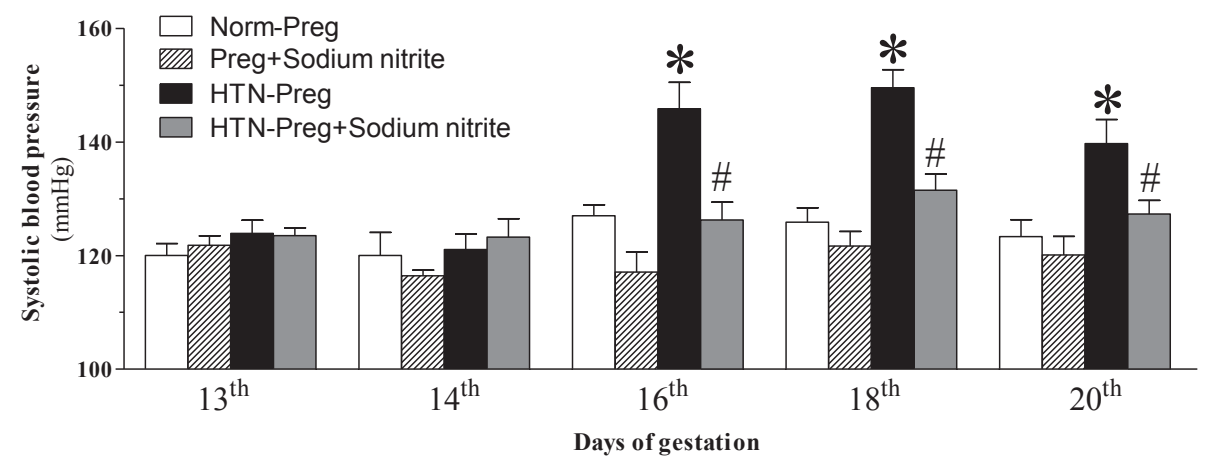

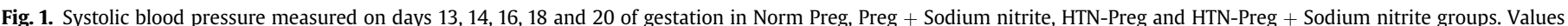

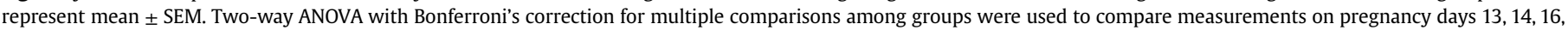

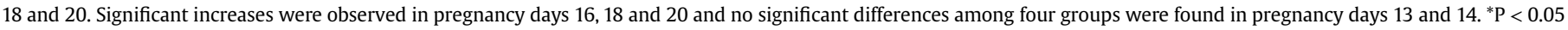
for HTN-Preg vs. Norm Preg and Preg + Sodium nitrite groups. \#P $<0.05$ for HTN-Preg + Sodium nitrite vs. HTN-Preg group.

NO may possibly be the driving force behind the maternal and fetal beneficial effects observed here.

Pregnancy-induced hypertension results in a state of endothelial dysfunction in preeclampsia [28,29], in which the exact mechanisms leading to the hypertension remain obscure. However, recent studies have proposed that there may be a pro/antiangiogenic imbalance in the pathogenesis of this disorder, suggesting that high circulating levels of sFlt-1 in preeclamptic women may be associated with hypertension [30,31]. Nevertheless, the specific mechanisms linking high sFlt-1 levels to hypertension are unclear. Earlier findings have shown reductions of bioavailable NO in preeclamptic women [8,9,32-34] and that endogenous NO production was reduced in $70 \%$ in the hypertensive response caused by the infusion of sFlt-1 into pregnant rats [35] and other animal models of hypertensive-pregnancy [10]. Thus, the present findings are in accordance to the hypothesis that increased plasma levels of sFlt-1 may be due to reductions of NO, suggesting that there may be a crosstalk between reduced NO synthesis and increases in sFlt-1 levels.

To test this hypothesis, pregnant rats were treated with the NOS inhibitor L-NAME and received sodium nitrite (or saline) by gavage. Upon chronic NOS inhibition, sodium nitrite reduces systolic blood pressure and blunts the increases in circulating sFlt- 1 levels. The present results are in accordance to previous studies suggesting that nitrite-derived NO after oral treatment with sodium nitrite may restore the vasodilator actions of the NO, resulting in antihypertensive effects in different rat models of hypertension [11-13,18,19,22,36-39]. Thus, our data suggests that nitritederived NO blunts the increases of sFlt- 1 and attenuates the hypertension-in-pregnancy induced by L-NAME.

The formation of NO from nitrite may occur by enzymatic or non-enzimatic pathways. The enzymatic pathways involve deoxyhaemoglobin [38], xanthine oxidase [40], myoglobin [41], aldehyde oxidase [42], mitochondrial cytochromes [43] and carbonic anhydrase [44], while the non-enzymatic pathway comprises the intragastric formation through reduction of nitrite to $\mathrm{NO}$ in low $\mathrm{pH}$ in the stomach $[13,37]$.

In our hands, following the treatment with sodium nitrite in pregnant rats without L-NAME (Preg + Sodium nitrite group) we observed increases in NO levels with no changes in systolic blood pressure, corroborating with previous studies that suggested compensatory mechanisms of normal blood pressure regulation $[19,22,45]$. Furthermore, we found increases in fetal weight with no changes in placental weight in the same Preg + Sodium nitrite group, which suggest nitrite-derived NO may afford better fetal maturational processes during late gestation $[15,35,46]$.
While treatment with L-NAME (HTN-Preg group) produced reductions in litter size and viable fetuses with more resorbed fetuses and reductions in placental weights without affecting the fetal weight, treatment with sodium nitrite was able to prevent these detrimental changes to the fetuses and placenta. We then suggest that the antihypertensive effects with concomitant increases in litter size and placental weight, with more viable and less resorbed fetuses may reflect the improvement of the uteroplacental perfusion caused by nitrite-derived NO $[47,48]$ and that NO may promote vasodilation of uteroplacental circulation [49,50]. Accordingly, earlier evidence has showed that the number of pups and placental weight are strongly correlated with uteroplacental blood flow and that reductions in these parameters may reflect the uteroplacental ischemia/hypoxia induced by L-NAME in rats [51]. Thus, we suggest that ischemic uteroplacental vasculature may be the ideal biochemical environment for the in vivo reduction of nitrite to NO.

We found higher circulating levels of VEGF in hypertension-inpregnancy induced by L-NAME than in normotensive pregnant rats treated with (or without) sodium nitrite (Norm-Preg and Preg + Sodium nitrite groups). Supporting our results, recent reports have demonstrated that increases in circulating levels of VEGF may result in adverse maternal and fetal side effects [31,52-55]. Accordingly, the exogenous VEGF administration in pregnant rats caused hypertension and hypercoagulation in the placental circulation, similar to human preeclampsia [56,57]. Additionally, mice with VEGF-overexpression revealed increases in maternal serum levels of sFlt-1, which resulted in pregnancy losses, placental vascular defects, increases in the number of resorption sites and decreases in the number of viable fetuses and preeclampsia-like symptoms including hypertension in the mother [56]. Thus, we may suggest that VEGF production could be elevated due to an increase in vascular shear stress, mainly in uteroplacental circulation [58]. Importantly, treatment of hypertensive pregnant rats with sodium nitrite (HTN-Preg + Sodium nitrite group) resulted in a reduction in VEGF levels. This may occur due to the fact that treatment with sodium nitrite may result in vasodilation [59], leading to decreases in vascular shear stress, and consequently reducing the production of VEGF [58], and that along with reductions of sFlt- 1 could be responsible for protective effects in mother and fetal side of hypertension-in-pregnancy induced by LNAME.

Additionally, the antioxidant effect of sodium nitrite demonstrated previously [11,19] is further supported by our results showing that sodium nitrite restored the plasma antioxidant capacity (higher TEAC in HTN-Preg + Sodium nitrite compared to HTN-Preg group). However, although there was no reduction of 

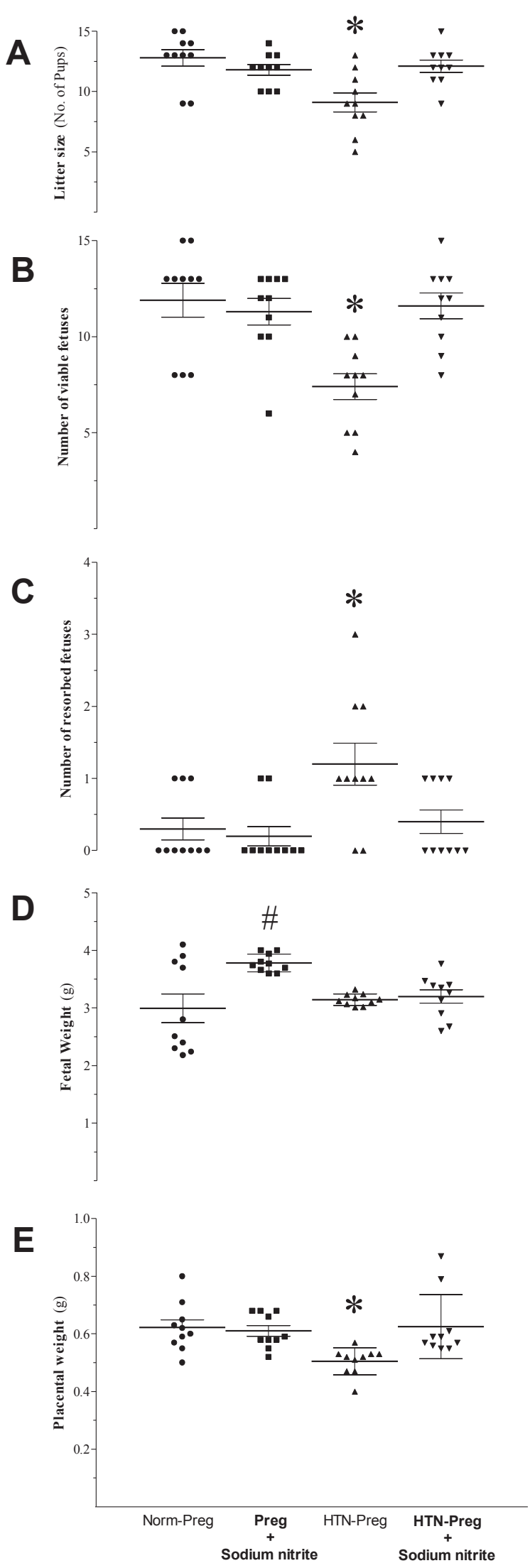

Fig. 2. Average of fetal and placental parameters were recorded on gestational-day 21: litter size (A), number of viable (B), resorbed (C) fetuses, fetal (D) and placental (E) weights in Norm Preg, Preg + Sodium nitrite, HTN-Preg and HTN-Preg + Sodium nitrite groups. Values represent mean \pm SEM. One-way ANOVA followed by Bonferroni's plasma antioxidant status in HTN-Preg compared to Norm-Preg group (presenting MTT assay with similar values), sodium nitrite treated (Preg + Sodium nitrite and HTN-Preg + Sodium nitrite) groups presented MTT assay with higher values than those obtained with Norm-Preg and HTN-Preg groups. In this regard, while we found these results with MTT assay to be intriguing, the sensitivity of methods used may be related to these apparently conflicting data. In fact, although the MTT assay is widely used to measure antioxidant status of plasma, the reference range and linearity for plasma antioxidant capacity measured with TEAC assay may be better than those found with MTT method $[26,27,60]$. Accordingly, a previous study showed that human plasma albumin is responsible for only $40-60 \%$ of the total MTT reduction observed and the platelets were not involved [27]. So, it is not entirely known which of the remaining plasma components might be responsible for the further direct reduction of MTT [27]. On the other hand, TEAC assay can determine the antioxidative effects of bilirubin, vitamin C, uric acid, polyphenols, and several proteins, including albumin; thus, the range for plasma antioxidant capacity measured with TEAC seems to be higher than that for the MTT method, since the antioxidative effect of proteins is accounted for, providing more sensitivity for determining the global antioxidant function [26]. Furthermore, it has been suggested that an oxidant/antioxidant imbalance may be associated with pregnancy complications [61] and that reductions of availability of endogenous NO during hypertension may be due to oxidative stress [61]. In fact, reactive oxygen species may result in the inactivation/sequestration of NO [62]. For these reasons, we then have decided to assess the circulating levels of MPO in our rat model of hypertension-in-pregnancy, since MPO is a possible source of reactive oxygen species that is also elevated in preeclamptic women $[63,64]$. thus, helping us to clarify the interaction between oxidative stress and hypertension-inpregnancy induced by L-NAME.

We have found that L-NAME increased the circulating levels of MPO in hypertensive pregnant rats treated with (or without) sodium nitrite (HTN-Preg + Sodium nitrite and HTN-Preg groups). This is particularly important due to the fact that MPO is an enzyme abundantly expressed in neutrophil granulocytes and that may become sequestered in the sub-endothelial space and accumulate in the cell matrix, releasing reactive oxygen species into circulation [65]. Corroborating these findings, studies have shown that members of the family heme-peroxidases, in which MPO is prototype, are able to consume NO, affecting its vasodilatatory response and exacerbating endothelial dysfunction [66]. Together, the protective (antihypertensive and antioxidant) effects of sodium nitrite treatment were found even under presence of increased MPO levels (similar levels in both HTN-Preg and HTN-Preg + Sodium nitrite groups), suggesting that the antioxidant effects of sodium nitrite presented no relation with MPO; however, the protective effects may result of inhibition of NADPH oxidase and xanthine oxidoreductase activities by sodium nitrite, preventing enzymesdependent reactive oxygen species production, as previously suggested [11,19].

The oxidative stress may be a common feature, even during healthy pregnancies, since there is an increase in the metabolic demand requested by maternal-fetal interface, which is offset by increases in antioxidant capacity [62,67]. However, upon

correction for multiple comparisons were used to compare measurements among four groups. Since only fetal weight in Norm-Preg group presented no normal distribution, the Mann-Whitney test was used to compare the fetal weight among the four groups. ${ }^{*} \mathrm{P}<0.05$ for HTN-Preg vs. Norm Preg, Preg + Sodium nitrite and HTN-Preg + Sodium nitrite groups. \#P $<0.05$ for Preg + Sodium nitrite vs. Norm Preg, HTN-Preg and HTNPreg + Sodium nitrite groups. 

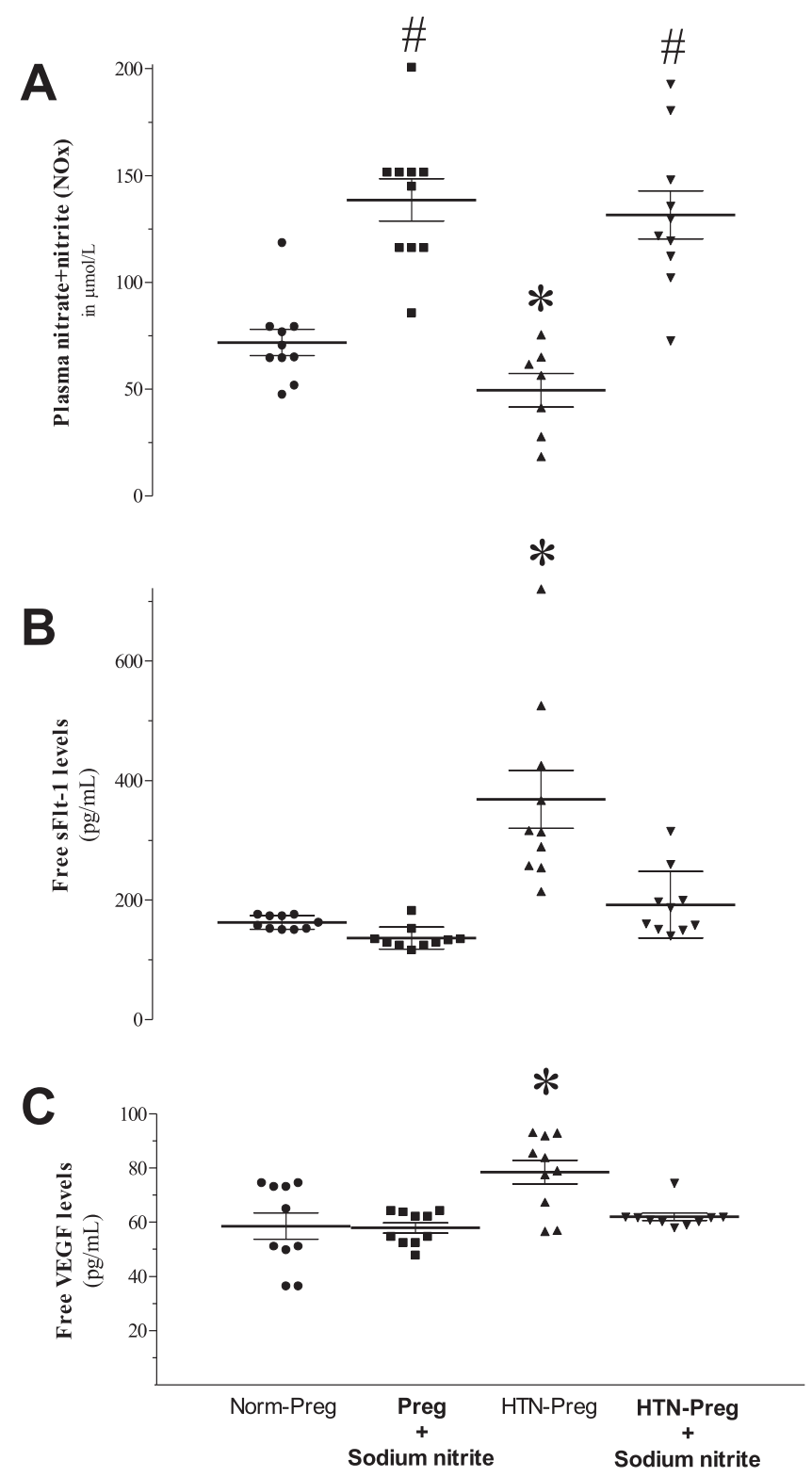

Fig. 3. Plasma NOx (A), circulating free sFlt-1 (B) and VEGF (C) levels in Norm Preg, Preg + Sodium nitrite, HTN-Preg and HTN-Preg + Sodium nitrite groups. Values represent mean \pm SEM. One-way ANOVA followed by Bonferroni's correction for multiple comparisons were used to compare measurements among four groups. ${ }^{*} \mathrm{P}<0.05$ for HTN-Preg vs. Norm Preg, Preg + Sodium nitrite and HTN-Preg + Sodium nitrite groups. \#P $<0.05$ for Preg + Sodium nitrite and HTN-Preg + Sodium nitrite vs. Norm Preg and HTN-Preg groups.

preeclampsia, antioxidant function has failed to prevent the damage promoted by excessive oxidative stress [68]. In this sense, maintaining antioxidant function may help in the prevention of injuries caused by oxidative stress during hypertension-inpregnancy. Combined, these findings provide evidence that the oral administration of sodium nitrite consistently decreases the blood pressure in association with major antioxidant effects in experimental hypertension $[11,19,45]$, including hypertension-inpregnancy induced by L-NAME. Thus, the antioxidant function of plasma maintained with sodium nitrite treatment may highlight the importance of further investigation in preeclamptic women, since counterbalancing the excessive oxidative damage may provide a great benefit in this related hypertensive disorders of pregnancy.
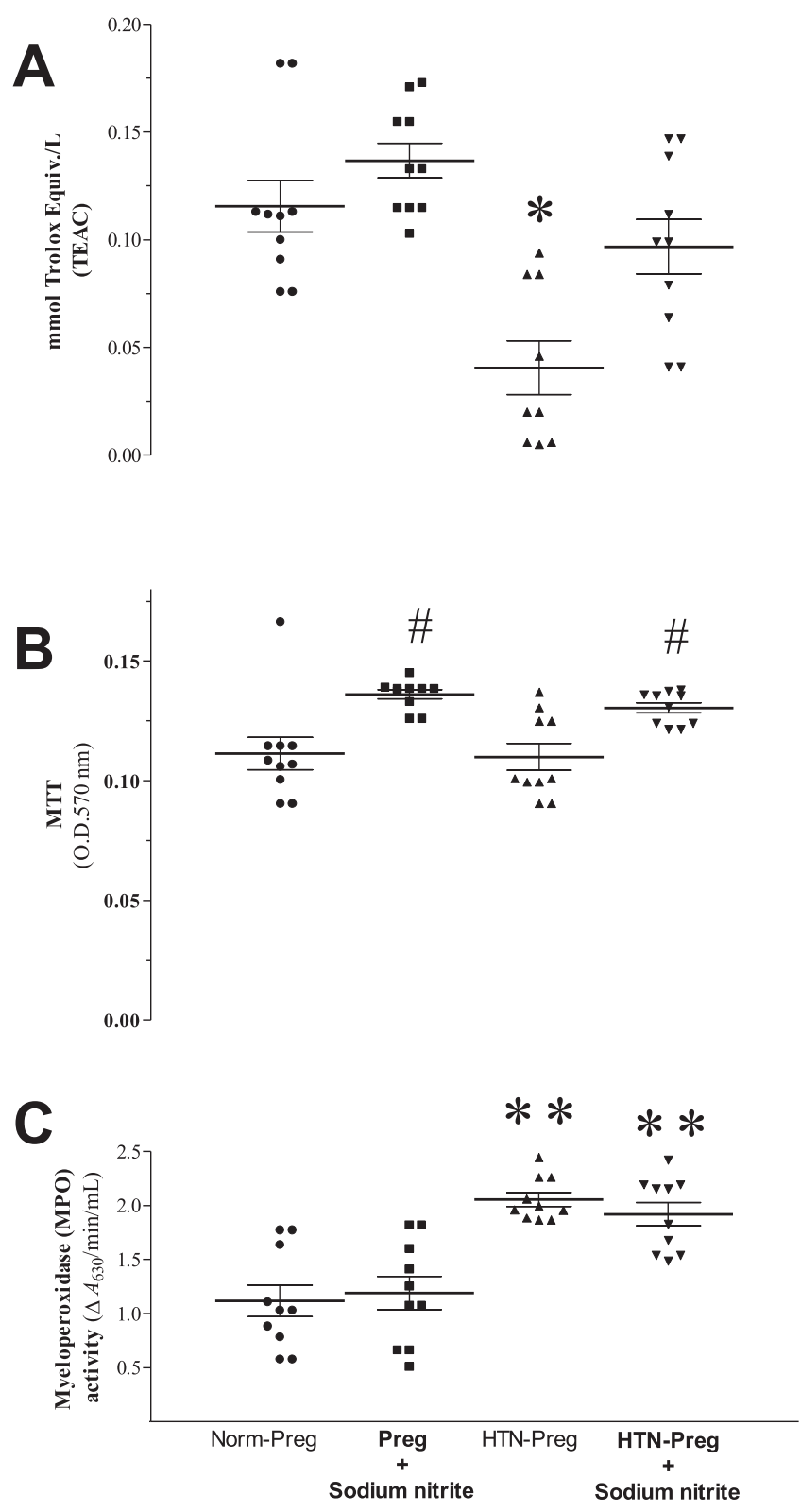

Fig. 4. Plasma antioxidant capacity (TEAC, A), antioxidant status (MTT, B) and MPO activity (C) in Norm Preg, Preg + Sodium nitrite, HTN-Preg and HTN-Preg + Sodium nitrite groups. Values represent mean + SEM. One-way ANOVA followed by Bonferroni's correction for multiple comparisons were used to compare measurements among four groups. ${ }^{*} \mathrm{P}<0.05$ for HTN-Preg vs. Norm Preg, Preg + Sodium nitrite and HTN-Preg + Sodium nitrite groups. \#P $<0.05$ for Preg + Sodium nitrite and HTNPreg + Sodium nitrite vs. Norm Preg and HTN-Preg groups. ${ }^{* *} \mathrm{P}<0.05$ for HTN-Preg and HTN-Preg + Sodium nitrite vs. Norm Preg and Preg + Sodium nitrite groups.

The present study has some limitations that should be taken into consideration. Firstly, although previous findings have shown that $80 \mathrm{mg} /$ day of sodium nitrite orally administrated was safe and well tolerated in healthy adults with few side effects, and no difference in methemoglobin levels compared to placebo [69], the methemoglobin levels could be a reason for concern in patients as a result of sodium nitrite treatment, particularly when sodium nitrite is used at high doses. Secondly, as clinical studies in obstetric patients always remain a delicate ethical issue, animal models serve to answer specific questions regarding the pathophysiology of preeclampsia. Moreover, comparability of data from clinical studies and experimental models always remains speculative [70]. Thirdly, 
the fetal weight should be interpreted with caution, since many factors may influence this parameter [71]. Finally, further studies are necessary to address the potential therapeutic of sodium nitrite during preeclampsia.

\section{Conclusion}

Taken together, our results suggest that nitrite-derived NO prevents hypertension-in-pregnancy induced by L-NAME and concomitantly reduces circulating plasma sFlt-1 and VEGF levels, improving the placental weight and litter size with more viable and less resorbed fetuses. In addition, our findings confirm the hypothesis that treatment with sodium nitrite attenuates the hypertension-in-pregnancy and that nitrite-derived NO may possibly be the driving force behind the maternal and fetal beneficial effects observed in the present study.

\section{Author contributions}

V.H.G.R., J.S.P.V., T.U.S.G. and R.A.N. performed experimental procedures, statistical analyses and contributed to discussion and revision of manuscript. C.A.D.J. contributed to statistical analyses and reviewed the discussion, edited the manuscript and gave final approval of the version to be published.

\section{Conflict of interest}

There are no known conflicts of interest.

\section{Acknowledgements}

This study was supported by funding from the Fundacao de Amparo a Pesquisa do Estado de Sao Paulo (FAPESP, Brazil). The following are gratefully acknowledged: Valeria Cristina Sandrim and Helio Kushima for their co-operation and advice with technician issues.

\section{References}

[1] J. Chang, L.D. Elam-Evans, C.J. Berg, J. Herndon, L. Flowers, K.A. Seed, C.J. Syverson, Pregnancy-related mortality surveillance-United States, 19911999, MMWR Surveill. Summ. 52 (2) (2003) 1-8.

[2] J. Uzan, M. Carbonnel, O. Piconne, R. Asmar, J.M. Ayoubi, Pre-eclampsia: pathophysiology, diagnosis, and management, Vasc. Health Risk Manag. 7 (2011) 467-474.

[3] T. Chaiworapongsa, P. Chaemsaithong, L. Yeo, R. Romero, Preeclampsia part 1: current understanding of it is pathophysiology, Nat. Rev. Nephrol. 10 (2014) 466-480.

[4] M. Noris, N. Perico, G. Remuzzi, Mechanisms of disease: pre-eclampsia, Nat. Clin. Pract. Nephrol. 1 (2) (2005) 98-114.

[5] R.J. Levine, S.E. Maynard, C. Qian, K.H. Lim, L.J. England, K.F. Yu E.F. Schisterman, R. Thadhani, B.P. Sachs, F.H. Epstein, B.M. Sibai, V.P. Sukhatme, S.A. Karumanchi, Circulating angiogenic factors and the risk of preeclampsia, N. Engl. J. Med. 350 (2004) 672-683.

[6] A.C. Palei, F.T. Spradley, J.P. Warrington, E.M. George, J.P. Granger, Pathophysiology of hypertension in pre-eclampsia: a lesson in integrative physiology, Acta Physiol. (Oxf) 208 (3) (2013) 224-233.

[7] R. Thadhani, T. Kisner, H. Hagmann, V. Bossung, S. Noack, W. Schaarschmidt, A. Jank, A. Kribs, O.A. Cornely, C. Kreyssig, L. Hemphill, A.C. Rigby, S. Khedkar T.H. Lindner, P. Mallmann, H. Stepan, S.A. Karumanchi, T. Benzing, Pilot study of extracorporeal removal of soluble fms-like tyrosine kinase 1 in preeclampsia, Circulation 124 (8) (2011) 940-950.

[8] V.C. Sandrim, A.C. Palei, I.F. Metzger, J.A. Gomes, R.C. Cavalli, J.E. Tanus-Santos, Nitric oxide formation is inversely related to serum levels of antiangiogenic factors soluble fms-like tyrosine kinase- 1 and soluble endogline in preeclampsia, Hypertension 52 (2) (2008) 402-407.

[9] A.M. Pimentel, N.R. Pereira, C.A. Costa, G.E. Mann, V.S. Cordeiro, R.S. Moura T.M. Brunini, A.C. Mendes-Ribeiro, A.C. Resende, L-arginine-nitric oxide pathway and oxidative stress in plasma and platelets of patients with preeclampsia, Hypertens. Res. 36 (9) (2013) 783-788.

[10] L.M. Amaral, D.C. Cornelius, A. Harmon, J. Moseley, J.N. Martin Jr., B. LaMarca, 17-hydroxyprogesterone caproate significantly improves clinical characteristics of preeclampsia in the reduced uterine perfusion pressure rat model,
Hypertension 65 (1) (2015) 225-231.

[11] M.F. Montenegro, J.H. Amaral, L.C. Pinheiro, E.K. Sakamoto, G.C. Ferreira, R.I. Reis, D.M. Marcal, R.P. Pereira, J.E. Tanus-Santos, Sodium nitrite downregulates vascular NADPH oxidase and exerts antihypertensive effects in hypertension, Free Radic. Biol. Med. 51 (1) (2011) 144-152.

[12] J.O. Lundberg, Nitric oxide metabolites and cardiovascular disease markers, mediators, or both? J. Am. Coll. Cardiol. 47 (3) (2006) 580-581.

[13] L.C. Pinheiro, M.F. Montenegro, J.H. Amaral, G.C. Ferreira, A.M. Oliveira, J.E. Tanus-Santos, Increase in gastric $\mathrm{pH}$ reduces hypotensive effect of oral sodium nitrite in rats, Free Radic. Biol. Med. 53 (4) (2012) 701-709.

[14] A.J. Mayr, W. Lederer, H.J. Wolf, M. Dünser, K. Pfaller, M.G. Mörtl, Morphologic changes of the uteroplacental unit in preeclampsia-like syndrome in rats, Hypertens. Pregnancy 24 (1) (2005) 29-37.

[15] C. Motta, C. Grosso, C. Zanuzzi, D. Molinero, N. Picco, R. Bellingeri, F. Alustiza, C. Barbeito, A. Vivas, M.C. Romanini, Effect of sildenafil on pre-eclampsia-like mouse model induced by L-name, Reprod. Domest. Anim. 50 (4) (2015) 611-616.

[16] A. Rajakumar, H.M. Michael, P.A. Rajakumar, E. Shibata, C.A. Hubel, S.A. Karumanchi, R. Thadhani, M. Wolf, G. Harger, N. Markovic, Extra-placental expression of vascular endothelial growth factor receptor-1 (Flt-1) and soluble Flt-1 (sFlt-1) by peripheral blood mononuclear cells (PBMCs) in normotensive and preeclamptic pregnant women, Placenta 26 (7) (2005) 563-573.

[17] X. Yang, L. Guo, X. Sun, X. Chen, X. Tong, Protective effects of hydrogen-rich saline in preeclampsia rat model, Placenta 32 (9) (2011) 681-686.

[18] L.C. Pinheiro, J.H. Amaral, G.C. Ferreira, M.F. Montenegro, G.H. Oliveira-Paula, J.E. Tanus-Santos, The antihypertensive effects of sodium nitrite are not associated with circulating angiotensin converting enzyme inhibition, Nitric Oxide 40 (2014) 52-59.

[19] J.H. Amaral, G.C. Ferreira, L.C. Pinheiro, M.F. Montenegro, J.E. Tanus-Santos, Consistent antioxidant and antihypertensive effects of oral sodium nitrite in DOCA-salt hypertension, Redox Biol. 5 (2015) 340-346.

[20] H.G. Classen, C. Stein-Hammer, H. Thöni, Hypothesis: the effect of oral nitrite on blood pressure in the spontaneously hypertensive rat. Does dietary nitrate mitigate hypertension after conversion to nitrite? J. Am. Coll. Nutr. 9 (5) (1990) 500-502.

[21] L.C. Pinheiro, J.H. Amaral, G.C. Ferreira, R.L. Portella, C.S. Ceron, M.F. Montenegro, J.C. Toledo Jr., J.E. Tanus-Santos, Gastric S-nitrosothiol formation drives the antihypertensive effects of oral sodium nitrite and nitrate in a rat model of renovascular hypertension, Free Radic. Biol. Med. 87 (2015) $252-562$.

[22] V.H. Gonçalves-Rizzi, R.A. Nascimento, J.S. Possomato-Vieira, C.A. Dias-Junior, Sodium nitrite prevents both reductions in circulating nitric oxide and hypertension in 7-day lead-treated rats, Basic Clin Pharmacol, Toxicol 118 (3) (2015) 225-230.

[23] R.Q. Ma, M.N. Sun, Z. Yang, Effects of preeclampsia-like symptoms at early gestational stage on feto-placental outcomes in a mouse model, Chin. Med. J. 123 (6) (2010) 707-712.

[24] C.A. Dias-Junior, E.M. Neto-Neves, M.F. Montenegro, J.E. Tanus-Santos, Hemodynamic effects of inducible nitric oxide synthase inhibition combined with sildenafil during acute pulmonary embolism, Nitric Oxide 23 (4) (2010) 284-288.

[25] K. Suzuki, H. Ota, S. Sasagawa, T. Sakatani, T. Fujikura, Assay method for myeloperoxidase in human polymorphonuclear leukocytes, Anal. Biochem. 132 (2) (1983) $345-352$.

[26] O. Erel, A novel automated direct measurement method for total antioxidant capacity using a new generation, more stable ABTS radical cation, Clin. Biochem. 37 (4) (2004) 277-285.

[27] L.O. Medina, C.A. Veloso, E. Abreu Borges, C.A. Isoni, M.R. Calsolari, M.M. Chaves, J.A. Nogueira-Machado, Determination of the antioxidant status of plasma from type 2 diabetic patients, Diabetes Res. Clin. Pract. 77 (2) (2007) $193-197$.

[28] L.J. Brennan, J.S. Morton, S.T. Davidge, Vascular dysfunctions in preeclampsia, Microcirculation 21 (1) (2014) 4-14.

[29] B. Lamarca, Endothelial dysfunction. An important mediator in the pathophysiology of hypertension during pre-eclampsia, Minerva Ginecol. 64 (4) (2012) 309-320.

[30] A. Luttun, P. Carmeliet, Soluble VEGF receptor Flt1: the elusive preeclampsia factor discovered? J. Clin. Investigation 111 (2003) 649-658.

[31] V. Tsatsaris, F. Goffin, C. Munaut, J.F. Brichant, M.R. Pignon, A. Noel, J.P. Schaaps, D. Cabrol, F. Frankenne, J.M. Foidart, Overexpression of the soluble vascular endothelial growth factor receptor in preeclamptic patients: pathophysiological consequences, J. Clin. Endocrinol. Metab. 88 (11) (2003) $5555-5563$.

[32] R.M. Ehsanipoor, W. Fortson, L.E. Fitzmaurice, W.X. Liao, D.A. Wing, D.B. Chen, K. Chan, Nitric oxide and carbon monoxide production and metabolism in preeclampsia, Reprod. Sci. 20 (5) (2013) 542-548.

[33] B. Schiessl, C. Strasburger, M. Bidlingmaier, I. Mylonas, U. Jeschke, F. Kainer, K. Friese, Plasma and urine concentrations of nitrite/nitrate and cyclic Guanosinemonophosphate in intrauterine growth restricted and preeclamptic pregnancies, Arch. Gynecol. Obstet. 274 (3) (2006) 150-154.

[34] N.M. Eleuterio, A.C. Palei, J.S. Rangel Machado, J.E. Tanus-Santos, R.C. Cavalli, V.C. Sandrim, Relationship between adiponectin and nitrite in healthy and preeclampsia pregnancies, Clin. Chim. Acta 423 (2013) 112-115.

[35] S.R. Murphy, B. LaMarca, K. Cockrell, M. Arany, J.P. Granger, L-arginine supplementation abolishes the blood pressure and endothelin response to 
chronic increases in plasma sFlt-1 in pregnant rats, Am. J. Physiol. Regul. Integr. Comp. Physiol. 302 (2) (2012) 259-263.

[36] M.F. Montenegro, L.C. Pinheiro, J.H. Amaral, G.C. Ferreira, R.L. Portella, J.E. Tanus-Santos, Vascular xanthine oxidoreductase contributes to the antihypertensive effects of sodium nitrite in l-NAME hypertension, Naunyn Schmiedeb. Arch. Pharmacol. 387 (6) (2014) 591-598.

[37] J.O. Lundberg, E. Weitzberg, J.M. Lundberg, K. Alving, Intragastric nitric oxide production in humans: measurements in expelled air, Gut 35 (11) (1994) $1543-1546$.

[38] K. Cosby, K.S. Partovi, J.H. Crawford, R.P. Patel, C.D. Reiter, S. Martyr, B.K. Yang, M.A. Waclawiw, G. Zalos, X. Xu, K.T. Huang, H. Shields, D.B. Kim-Shapiro, A.N. Schechter, R.O. Cannon 3rd, M.T. Gladwin, Nitrite reduction to nitric oxide by deoxyhemoglobin vasodilates the human circulation, Nat. Med. 9 (12) (2003) 1498-1505.

[39] S.M. Ghosh, V. Kapil, I. Fuentes-Calvo, K.J. Bubb, V. Pearl, A.B. Milsom, R. Khambata, S. Maleki-Toyserkani, M. Yousuf, N. Benjamin, A.J. Webb, M.J. Caulfield, A.J. Hobbs, A. Ahluwalia, Enhanced vasodilator activity of nitrite in hypertension: critical role for erythrocytic xanthine oxidoreductase and translational potential, Hypertension 61 (2013) 1091-1102.

[40] P. Tripatara, N.S.A. Patel, A. Webb, K. Rathod, F.M.J. Lecomte, E. Mazzon, S. Cuzzocrea, M.M. Yaqoob, A. Ahluwalia, C. Thiemermann, Nitrite-derived nitric oxide protects the rat kidney against ischemia/reperfusion injury in Vivo: role for xanthine oxidoreductase, J. Am. Soc. Nephrol. 28 (2007) $570-580$.

[41] S. Shiva, Z. Huang, R. Grubina, J. Sun, R.A. Ringwood, P.H. MacArthur, X. Xu, E. Murphy, V.M. Darley-Usmar, M.T. Gladwin, Deoxymyoglobin is a nitrite reductase that generates nitric oxide and regulates mitochondrial respiration, Circ. Res. 100 (5) (2007) 654-661.

[42] H. Li, H. Cui, T.K. Kundu, W. Alzawahra, J.L. Zweier, Nitric oxide production from nitrite occurs primarily in tissues not in the blood: critical role of xanthine oxidase and aldehyde oxidase, J. Biol. Chem. 283 (26) (2008) $17855-17863$.

[43] A.V. Kozlov, K. Staniek, H. Nohl, Nitrite reductase activity is a novel function of mammalian mitochondria, FEBS Lett. 454 (1999) 127-130.

[44] R. Aamand, T. Dalsgaard, F.B. Jensen, U. Simonsen, A. Roepstorff, A. Fago, Generation of nitric oxide from nitrite by carbonic anhydrase: a possible link between metabolic activity and vasodilation, Am. J. Physiol. Heart Circ. Physiol. 297 (6) (2009) 2068-2074.

[45] M.F. Montenegro, L.C. Pinheiro, J.H. Amaral, D.M. Marçal, A.C. Palei, A.J. CostaFilho, J.E. Tanus-Santos, Antihypertensive and antioxidant effects of a single daily dose of sodium nitrite in a model of renovascular hypertension, Naunyn Schmiedeb. Arch. Pharmacol. 385 (5) (2012) 509-517.

[46] E.E. Gillis, J.N. Mooney, M.R. Garrett, J.P. Granger, J.M. Sasser, Sildenafil treatment ameliorates the maternal syndrome of preeclampsia and rescues fetal growth in the dahl salt-sensitive rat, Hypertension 67 (3) (2016) 647-653.

[47] S.R. Ramesar, I. Mackraj, P. Gathiram, J. Moodley, Sildenafil citrate improves fetal outcomes in pregnant, L-NAME treated, Sprague-Dawley rats, Eur. J. Obstet. Gynecol. Reprod. Biol. 149 (1) (2010) 22-26.

[48] J.O. Lundberg, M.T. Gladwin, E. Weitzberg, Strategies to increase nitric oxide signalling in cardiovascular disease, Nat. Rev. Drug Discov. 14 (9) (2015) 623-641.

[49] L. Schaffer, J. Vogel, C. Breymann, M. Gassmann, H.H. Marti, Preserved placental oxygenation and development during severe systemic hypoxia, Am. J. Physiol. Regul. Integr. Comp. Physiol. 290 (3) (2006) 844-851.

[50] M.C. Pustovrh, A. Jawerbaum, V. White, E. Capobianco, R. Higa, N. Martınez, J.J. Lopez-Costa, E. Gonzalez, The role of nitric oxide on matrix metalloproteinase 2 (MMP2) and MMP9 in placenta and fetus from diabetic rats, Reproduction 134 (4) (2007) 605-613.

[51] A. Kaya, A. Boztosun, H. Seckin, A.S. Guven, Z. Kucukdurmaz, S. Gulturk, O. Cevit, The evaluation of hypoxia-inducible factor 1 in N-nitro-L-arginine methyl ester preeclampsia model of pregnant rats, J. Investig. Med. 59 (8) (2011) 1268-1272.
[52] C.R. Ozawa, A. Banfi, N.L. Glazer, G. Thurston, M.L. Springer, P.E. Kraft, D.M. McDonald, H.M. Blau, Microenvironmental VEGF concentration, not tota dose, determines a threshold between normal and aberrant angiogenesis, J. Clin. Invest 113 (4) (2004) 516-527.

[53] L. Miquerol, B.L. Langille, A. Nagy, Embryonic development is disrupted by modest increases in vascular endothelial growth factor gene expression, Development 127 (18) (2000) 3941-3946.

[54] S. Zhao, X. Gu, L.J. Groome, Y. Wang, Decreased nephrin and GLEPP-1, but increased VEGF, Flt-1, and nitrotyrosine, expressions in kidney tissue sections from women with preeclampsia, Reprod. Sci. 16 (10) (2009) 970-979.

[55] A. Hunter, M. Aitkenhead, C. Caldwell, G. McCracken, D. Wilson, N. McClure, Serum levels of vascular endothelial growth factor in preeclamptic and normotensive pregnancy, Hypertension 36 (6) (2000) 965-969.

[56] X. Fan, A. Rai, N. Kambham, J.F. Sung, N. Singh, M. Petitt, S. Dhal, R. Agrawal, R.E. Sutton, M.L. Druzin, S.S. Gambhir, B.K. Ambati, J.C. Cross, N.R. Nayak, Endometrial VEGF induces placental sFLT1 and leads to pregnancy complications, J. Clin. Invest 124 (11) (2014) 4941-4952.

[57] Y. Murakami, T. Kobayashi, K. Omatsu, M. Suzuki, R. Ohashi, T. Matsuura M. Sugimura, N. Kanayama, Exogenous vascular endothelial growth factor can induce preeclampsia-like symptoms in pregnant mice, Semin. Thromb. Hemost. 31 (3) (2005) 307-313.

[58] A.F. Tanbe, R.A. Khalil, Circulating and vascular bioactive factors during hypertension in pregnancy, Curr. Bioact. Compd. 6 (1) (2010) 60-75.

[59] A.L. Sindler, B.S. Fleenor, J.W. Calvert, K.D. Marshall, M.L. Zigler, D.J. Lefer, D.R. Seals, Nitrite supplementation reverses vascular endothelial dysfunction and large elastic artery stiffness with aging, Aging Cell 10 (3) (2011) 429-437.

[60] T. Mosmann, Rapid colorimetric assay for cellular growth and survival: application to proliferation and cytotoxicity assays, J. Immunol. Methods 65 $(1-2)(1983) 55-63$.

[61] K. Matsubara, T. Higaki, Y. Matsubara, A. Nawa, Nitric oxide and reactive oxygen species in the pathogenesis of preeclampsia, Int. J. Mol. Sci. 16 (3) (2015) 4600-4614.

[62] L.C. Sánchez-Aranguren, C.E. Prada, C.E. Riaño-Medina, M. Lopez, Endothelia dysfunction and preeclampsia: role of oxidative stress, Front. Physiology 5 (2014) 372 .

[63] R.E. Gandley, J. Rohland, Y. Zhou, E. Shibata, G.F. Harger, A. Rajakumar V.E. Kagan, N. Markovic, C.A. Hubel, Increased myeloperoxidase in the placenta and circulation of women with preeclampsia, Hypertension 52 (2) (2008) 387-393.

[64] J.R. Mellembakken, P. Aukrust, M.K. Olafsen, T. Ueland, K. Hestdal, V. Videm, Activation of leukocytes during the uteroplacental passage in preeclampsia, Hypertension 39 (1) (2002) 155-160.

[65] T.H. Hung, S.F. Chen, L.M. Lo, M.J. Li, Y.L. Yeh, T.T. Hsieh, Myeloperoxidase in the plasma and placenta of normal pregnant women and women with pregnancies complicated by preeclampsia and intrauterine growth restriction, Placenta 33 (4) (2012) 294-303.

[66] H.M. Abu-Soud, M.L. Hazen, Nitric oxide is a physiological substrate for mammalian peroxidases, J. Biol. Chem. 275 (48) (2000) 37524-37532.

[67] L. Myatt, R.P. Webster, Vascular biology of preeclampsia, J. Thrombosis Haemostasis 7 (3) (2009) 375-384.

[68] S.M. Ali, R.A. Khalil, Genetic, immune and vasoactive factors in the vascular dysfunction associated with hypertension in pregnancy, Expert Opin. Ther. Targets 19 (11) (2015) 1495-1515.

[69] A.E. DeVan, L.C. Johnson, F.A. Brooks, T.D. Evans, J.N. Justice, C. CruickshankQuinn, N. Reisdorph, N.S. Bryan, M.B. McQueen, J.R. Santos-Parker, M.B. Chonchol, C.J. Bassett, A.L. Sindler, T. Giordano, D.R. Seals, Effects of sodium nitrite supplementation on vascular function and related small metabolite signatures in middle-aged and older adults, J. Appl. Physiol. 120 (4) (1985) 416-425.

[70] F.P. McCarthy, J.C. Kingdom, L.C. Kenny, S.K. Walsh, Animals model of preeclampsia; uses and limitation, Placenta 32 (2011) 413-419.

[71] N.A. Norman, N.W. Bruce, Fetal and placental weight relationships in the rat at days 13 and 17 of gestation, J. Reprod. Fertil. 57 (2) (1979) 345-348. 